Series A

I. MATHEMATICA

440

\title{
ON NUMBERS WITH SMALL PRIME DIVISORS
}

BY

VEIKKO ENNOLA

H E L S I K I 1969

S U O M A L I N E N T E DEAKATEMIA

doi:10.5186/aasfm.1969.440 
Communicated 13 December 1968 by P. J. Myrberg and K. InkenI 


\section{On numbers with small prime divisors}

1. For $x>0, y \geqq 2$, let $\Psi(x, y)$ denote the number of positive integers not exceeding $x$ which contain no prime factors greater than $y$. Various estimates for $\Psi(x, y)$ have been given by several authors (see $[1],[2],[3],[5]$, and the further literature cited in these papers). The results of de Bruijn show how the asymptotic behaviour of $\Psi(x, y)$ changes around $y=\log x$. In this paper we investigate the case when $y$ is much smaller than $\log x$.

For $\operatorname{Re} s>0$, denote

$$
f^{\prime}(s)=\prod_{p \leqq y}\left(1-p^{-s}\right)^{-1}=\sum_{n}^{\prime} n^{-s},
$$

where, as throughout this paper, the notation $\Sigma^{\prime}$ indicates a sum over positive integers $n$ such that $p: n$ implies $p \leqq y$. By well known arguments, if $x$ is not an integer,

$$
\Psi(x, y)=\frac{1}{2 \pi i} \int_{\sigma-i \infty}^{\sigma+i \infty} x^{s} f(s) s^{-1} d s,
$$

where $\sigma>0$. Hence, as noted by de Bruijn [1], it can be expected that the residue of the integrand at $s=0$ will furnish a good approximation to $\Psi(x, y)$. For small values of $y$, we shall show that this is the case. Owing to some difficulties concerning the estimation of the coefficients in the Laurent series about $s=0$ of the function $f(s)$, we are able to prove the result only for $2 \leqq y \leqq(\log x)^{-)}$, where $\Theta<\frac{3}{4}$, but it can probably be extended to $\Theta<1$.

The method is to use integration with a suitable kernel. Our kernel $\exp \left(-\lambda w^{2}+i w\right)$, for $s=\sigma(1+i w)$, seems to be particularly serviceable in this case. Otherwise our method is in many respects similar to that of Ingham [4].

2. At first we consider a trivial case. Put

$$
Z=\log x, \quad N=\pi(y)=\sum_{p \leqq y} 1
$$


Now $\Psi(x, y)$ is the number of solutions of the inequality

$$
\sum_{i=1}^{N} u_{i} \log p_{i} \leqq Z
$$

in non-negative integers $u_{i}$, where the $p_{i}$ :s denote the primes $\leqq y$. Interpreting this as a question about the number of lattice points in a certain $N$-dimensional simplex, we easily find, by elementary geometrical considerations, that

$$
P(N !)^{-1} Z^{N} \leqq \Psi(x, y) \leqq P(N !)^{-1}(Z+q)^{N},
$$

where

$$
q=\sum_{i=1}^{N} \log p_{i} \sim y
$$

and

$$
P=\prod_{p \leqq y}(\log p)^{-1}
$$

Observing that $N \sim y(\log y)^{-1}$, we obtain*)

Theorem 1. For $2 \leqq y \leqq Z^{1 / 2}$, we have uniformly

$$
\Psi\left(e^{Z}, y\right)=P(N !)^{-1} Z^{N}\left(1+O\left(y^{2} Z^{-1}(\log y)^{-1}\right)\right) .
$$

3. We shall need some facts about integrals of the form

$$
J_{k}(\beta)=\int_{-\infty}^{\infty} \exp \left(-\lambda w^{2}+i \beta w\right)(1+i w)^{-k} d w,
$$

where $\lambda$ is a (small) positive constant to be chosen later, $\beta$ is real, and $k$ is a non-negative integer. We have

$$
J_{0}(\beta)=\pi^{1 / 2} \lambda^{-1 / 2} \exp \left(-\frac{\beta^{2}}{4 \lambda}\right) .
$$

We use the notation

$$
\operatorname{Erfc}(t)=\int_{i}^{\infty} \exp \left(-u^{2}\right) d u
$$

For $t>0$,

*) We would like to take this opportunity to point out an error in the paper of A. I. Vinogradov [5]. Our Theorem 1 clearly shows that the result stated as case 2) of Theorem 1 in [5] cannot be true. 
(5)

$$
\operatorname{Erfc}(t)<1 / 2 t^{-1} \exp \left(-t^{2}\right)
$$

Using integration by parts, we obtain

$$
J_{k}(\beta)=\frac{\beta-2 \lambda}{k-1} J_{k-1}(\beta)+\frac{2 \lambda}{k-1} J_{k-2}(\beta) \quad(k \geqq 2) .
$$

Since

$$
\frac{d}{d \beta} J_{1}(\beta)=J_{0}(\beta)-J_{1}(\beta),
$$

we find that $J_{1}(\beta)$ is a solution of the differential equation

$$
\frac{d Y}{d \beta}+Y=\pi^{1 / 2} \lambda^{-1 / 2} \exp \left(-\frac{\beta^{2}}{4 \lambda}\right)
$$

The general solution of $(7)$ is

$$
Y=C e^{-\beta}+2 \pi e^{\lambda-\beta}\left(1-\pi^{-1 / 2} \operatorname{Erfc}\left(\frac{\beta-2 \lambda}{2 \sqrt{\lambda}}\right)\right) .
$$

For $\beta \rightarrow-\infty$, the second term in (8) approaches zero, and, by Riemann's lemma, so does $J_{1}(\beta)$. Hence $C=0$, and we have

$$
J_{1}(\beta)=2 \pi e^{\lambda-\beta}\left(1-\pi^{-1 / 2} \operatorname{Erfc}\left(\frac{\beta-2 \lambda}{2 \sqrt{\lambda}}\right)\right) .
$$

Let $J_{k}=J_{k}(1) \quad(k=0,1, \ldots)$. Assuming that $\lambda$ is sufficiently small (e.g. $\lambda<\frac{1}{16}$ ), we have

and

$$
J_{0}<J_{1}
$$

$$
(1-2 \lambda) J_{1}<J_{2}<J_{1} .
$$

Furthermore, it is easy to see, by (6), that

$$
J_{k}<J_{k-1}
$$$$
(k \geqq 2) \text {. }
$$

Now (6) implies, for $k \geqq 3$,

$$
J_{k}>\frac{1}{k-1} J_{k-1},
$$

so that, by (10),

$$
J_{k}>\frac{1-2 \lambda}{(k-1) !} J_{1}
$$$$
(k \geqq 1) \text {. }
$$ 
On the other hand, for $3 \leqq k \leqq N+1$, we have

$$
J_{k-2}<\frac{k-2}{1-2 \lambda} J_{k-1}<\frac{N}{1-2 \lambda} J_{k-1}
$$

whence

$$
\begin{aligned}
J_{k} & <\frac{1}{k-1}\left(1+\frac{2 \lambda N}{1-2 \lambda}-2 \lambda\right) J_{k-1} \\
& <\frac{1+3 \lambda N}{k-1} J_{k-1} .
\end{aligned}
$$

By (10), this is also true for $k=2$. Hence we have

$$
J_{k}<\frac{(1+3 \lambda N)^{N}}{(k-1) !} J_{1} \quad(1 \leqq k \leqq N+1) .
$$

4. Let us now consider in detail the Laurent series about $s=0$ of the function $f(s)$. Put

$$
\frac{z}{1-e^{-z}}=\sum_{n=0}^{\infty} c_{n} z^{n} \quad(|z|<2 \pi)
$$

where

$$
c_{0}=1, \quad c_{1}=1 / 2, \quad c_{2 k+1}=0(k \geqq 1), \quad c_{2 k}=(-1)^{\mathrm{k}+1} \frac{B_{k}}{(2 k) !}(k \geqq 1),
$$

with

$$
B_{k}=2(2 k) !(2 \pi)^{-2 k} \zeta(2 k)
$$

Thus

$$
\left|c_{n}\right| \leqq 2^{-n} \quad(n=0,1,2, \ldots) .
$$

We have

$$
f(s)=P s^{-N} \prod_{p \leqq y} \sum_{n=0}^{\infty} c_{n} \log ^{n} p s^{n}=P s^{-N} \sum_{n=0}^{\infty} d_{n} s^{n},
$$

say, where $P$ is defined by (3), the series being convergent for

$$
|s|<2 \pi(\log y)^{-1} \text {. }
$$

From (14) we infer

$$
\left|d_{n}\right| \leqq\left(\begin{array}{c}
N+n-1 \\
n
\end{array}\right) 2^{-n} \log ^{n} y \quad(n=0,1,2, \ldots),
$$
where the binomial coefficient gives the number of solutions of $\sum_{i=1}^{N} u_{i}=n$
in non-negative integers $u_{i}$. We shall need the following 
Lemma. If $n \leqq N^{\frac{2}{3}}$ and $N$ is large enough, then

$$
d_{n} \underset{1 \leqq i_{1}<i_{2}<\cdots<i_{n} \leqq N}{2^{-n}} \log p_{i_{1}} \log p_{i_{2}} \ldots \log p_{i_{n}} .
$$

Proof. By definition, $d_{n}$ is the coefficient of $s^{n}$ in the product

$$
\prod_{p \leqq y} \sum_{k=0}^{\infty} c_{k} \log ^{k} p s^{k} .
$$

The right-hand side of (17) being the coefficient of $s^{n}$ in the product

$$
\prod_{p \leqq y}(1+1 / 2 \log p s)
$$

we must show that the total contribution to $d_{n}$ of all the other terms is non-negative.

Suppose that $\gamma s^{n}$, where

$$
\gamma=\prod_{i=1}^{N} c_{k_{i}} \log ^{k_{i}} p_{i},
$$

with $\sum_{i=1}^{N} k_{i}=n$, is any term in the product (18) containing $s^{n}$ such that at least one of the $k_{i}: \mathrm{s}$ is $\geqq 2$. Put

$$
\gamma_{0}=\prod_{\substack{i=1 \\ k_{i} \geqq 2}}^{N} c_{k_{i}} \log ^{k_{i}} p_{i} .
$$

We call $\gamma_{0}$ the $\gg$ support of $\gamma^{\prime}$. Write

$$
m=\sum_{\substack{i=1 \\ k_{i} \geqq 2}}^{N} k_{i} .
$$

Denote by $\mathcal{K}\left(\gamma_{0}\right)$ the set of all primes $\leqq y$ not appearing in (20). Let $C_{1}\left(\gamma_{0}\right)$ be the set of all those numbers $\gamma$ such that $\gamma s^{n}$ is a term in the product (18) containing $s^{n}$, which have the same support $\gamma_{0}$. Then

$$
\sum_{\gamma \in \mathcal{G}\left(\gamma_{0}\right)} \gamma=\gamma_{0}\left(\frac{1 / 2}{v_{1}<v_{2}<\cdots<v_{n-m}} \sum_{v_{1}} \log p_{v_{1}} \log p_{r_{2}} \ldots \log p_{v_{n-m}},\right.
$$

where the sum on the right-hand side is to be taken over all primes in K $\left(\gamma_{0}\right)$.

Suppose now that $\gamma$, written in the form (19), is negative. Then at least one of the $k_{i}$ :s must be divisible by 4 . Let $j$ be the first index for which this happens. Take

$$
\bar{\gamma}_{0}=\frac{c_{k_{j}-2}}{c_{k_{j}}} \gamma_{0}\left(\log p_{j}\right)^{-2} .
$$


Observing that

we have

$$
-\frac{c_{k_{j}-2}}{c_{k_{j}}}=4 \pi^{2} \frac{\zeta\left(k_{j}-2\right)}{\zeta\left(k_{j}\right)}>4 \pi^{2}
$$

$$
\bar{\gamma}_{0}>4 \pi^{2}\left|\gamma_{0}\right|(\log y)^{-2}
$$

Again

$$
\sum_{\gamma \in \bar{G}\left(\bar{\gamma}_{0}\right)} \gamma=\bar{\gamma}_{0}(1 / 2)^{n-m+2} \sum_{v_{1}<v_{2}<\ldots<v_{n-m+2}} \log p_{v_{1}} \log p_{v_{2}} \ldots \log p_{v_{n-m+2}},
$$

where the sum is also to be taken over all primes in $\mathcal{K}\left(\gamma_{0}\right)$. Let $K$ be the number of elements in $\mathcal{K}\left(\gamma_{0}\right)$. Clearly (20) can contain at most $1 / 2 m-1$ primes $p_{i}$, so that $K \geqq N-1 / 2 m+1$. Multiplying each product

$$
\log p_{v_{1}} \log p_{v_{2}} \ldots \log p_{v_{n-m}}
$$

in (21) by $\log p_{\varkappa} \log p_{\mu}$, where $p_{\varkappa}$ and $p_{\mu}$ are two different new primes belonging to $\mathcal{K}\left(\gamma_{0}\right)$, both $\geqq N^{1 / 2}$, we obtain terms such as in (22). The same new term will come out at most

$$
\left(\begin{array}{c}
n-m+2 \\
2
\end{array}\right)<1 / 2 n^{2}
$$

times. The number of choices is at least

$$
\left(\begin{array}{c}
K-(n-m)-N^{1 / 2} \\
2
\end{array}\right)>1 / 2\left(N-n-N^{1 / 2}\right)^{2}>1 / 2\left(N-2 N^{\frac{2}{3}}\right)^{2}>\frac{1}{4} N^{{ }^{2}}
$$

if $N$ is large enough. We also have

$$
\log p_{\varkappa} \log p_{\mu}>\frac{1}{4} \log ^{2} y \text {. }
$$

Combining these facts with (21) and (22), we infer

$$
\begin{aligned}
\sum_{\gamma \in \mathcal{G}\left(\bar{\gamma}_{0}\right)} \gamma & >4 \pi^{2}(\log y)^{-2} \frac{1}{4} \sum_{\gamma \in \mathcal{G}\left(\gamma_{0}\right)}|\gamma| \frac{1}{2} \frac{N^{2}}{n^{2}} \frac{1}{4} \log ^{2} y \\
& >\left(\frac{N}{n}\right)_{\gamma \varepsilon \overline{\mathcal{G}}\left(\gamma_{0}\right)}^{2}|\gamma| \geqq n \sum_{\gamma \varepsilon \mathcal{G}\left(\gamma_{0}\right)}|\gamma| .
\end{aligned}
$$

As the number of negative supports $\gamma_{0}$ corresponding to the same $\bar{\gamma}_{0}$ is clearly less than $n$, our lemma easily follows.

We define

$$
\begin{aligned}
& R(t)=\sum_{n=0}^{N} \frac{d_{n} t^{n}}{(N-n) !}, \\
& Q(t)=\prod_{p \leqq y}(1+1 / 2 N t \log p) .
\end{aligned}
$$


Then, in fact, $P \log ^{N} \times R\left((\log x)^{-1}\right)$ is the residue at $s=0$ of the integrand in (1), $P$ being defined by (3).

If $t>0$ and $t y$ is sufficiently small

$$
\exp \left(\frac{1}{4} N^{2} t\right)<Q(t)<\exp \left(1 / 2 N^{2} t \log y\right)
$$

and, especially,

$$
Q(t)=1+O\left(N^{2} t \log y\right) \quad \text { for } \quad 0<t \ll y^{-2} \log y
$$

Let

$$
0<t<N^{-(1 / \Theta)} \quad\left(0<\Theta<\frac{3}{4}\right)
$$

and put

$$
n_{0}=n_{0}(t)=e \log y \max \left\{1, N^{2} t\right\} .
$$

Then $n_{0} \leqq N^{2 / 3}$, if $N \geqq N_{0}(\Theta)$ is large enough. Our lemma is thus applicable for $0 \leqq n \leqq n_{0}$. For $n>n_{0}$ we have, by (16),

$$
\begin{aligned}
N ! \frac{\left|d_{n}\right| t^{n}}{(N-n) !} & \leqq\left(\begin{array}{c}
N+n-1 \\
n
\end{array}\right) 2^{-n} \log ^{n} y t^{n} \frac{N !}{(N-n) !} \\
& <N^{2 n}(n !)^{-1} 2^{-n} \log ^{n} y t^{n} \\
& <\frac{1}{2}\left(\frac{e N^{2} t \log y}{2 n}\right)^{n}<2^{-n-1},
\end{aligned}
$$

so that

$$
N ! \sum_{n_{0}<n \leqq N} \frac{d_{n} ! t^{n}}{(N-n) !}<(1 / 2)^{n_{0}}<y^{-1}
$$

Expanding $Q(t)$ in powers of $t$, we similarly obtain, for that part of this expansion which contains powers of $t$ greater than $n_{0}$, the estimate

$$
\sum_{n_{0}<n \leqq N}\left(\begin{array}{l}
N \\
n
\end{array}\right) N^{n} t^{n} 2^{-n} \log ^{n} y<(1 / 2)^{n_{0}}<y^{-1} .
$$

Observing that, for $0 \leqq n \leqq n_{0}$ and $N$ sufficiently large,

$$
\frac{N !}{(N-n) !} \geqq N^{n}\left(1-\frac{n}{N}\right)^{n}>N^{n} \exp \left(-2 n_{0}^{2} N^{-1}\right),
$$

we can conclude that under the assumption (25), for $N \geqq N_{0}(\Theta)$,

$$
N ! R(t)>Q(t) \exp \left(-2 n_{0}^{2} N^{-1}\right)-2 y^{-1} .
$$


5. For $\operatorname{Re} s=\sigma>0$, let

$$
F(s)=s^{-1} f(s)=\sum_{n}^{\prime} s^{-1} n^{-s} .
$$

Put $s=\sigma(1+i w)$, multiply both sides by $\sigma \exp \left(-\lambda w^{2}+i w\right)$ and integrate with respect to $w$ from $-\infty$ to $\infty$. In virtue of (9), one obtains

$$
\begin{aligned}
& 2 \pi e^{\lambda-1} \sum_{n}^{\prime}\left(1-\pi^{-1 / 2} \operatorname{Erfc}\left(\frac{1-\sigma \log n-2 \lambda}{2 \sqrt{\lambda}}\right)\right) \\
= & \int_{-\infty}^{\infty} \sigma \exp \left(-\lambda w^{2}+i w\right) F(\sigma(1+i w)) d w
\end{aligned}
$$

the inversion of the order of summation and integration being justified by absolute convergence. Using (5) we can immediately derive the following lower bound for the left-hand side of (29)

$$
2 \pi e^{\lambda-1}\left(1-\vartheta^{-1} \exp \left(-\vartheta^{2}\right)\right) \Psi\left(\exp \left(\sigma^{-1}\left(1-2 \lambda-2 \vartheta \lambda^{1 / 2}\right)\right), y\right),
$$

where $\vartheta$ is any positive number. As regards the upper bound, we see at first that the left-hand side of (29) is less than

$$
\begin{aligned}
& 2 \pi e^{\lambda-1} \Psi\left(\exp \left(\sigma^{-1}\left(1-2 \lambda+2 \vartheta \lambda^{1 / 2}\right)\right), y\right) \\
& +2 \pi^{1 / 2} e^{\lambda-1} \sum_{n>n_{1}}^{\prime} \operatorname{Erfc}\left(\frac{\sigma \log n+2 \lambda-1}{2 \sqrt{\lambda}}\right),
\end{aligned}
$$

where again $\vartheta$ is any positive number and $n_{1}=\exp \left(\sigma^{-1}\left(1-2 \lambda+2 \vartheta \lambda^{1 / 2}\right)\right)$. Instead of $n$, define a new variable $u$ by

$$
2 \lambda^{1 / 2} u=\sigma \log n+2 \lambda-1
$$

and write

$$
\Phi(u)=\Psi\left(\exp \left(\sigma^{-1}\left(1-2 \lambda+2 u \lambda^{1 / 2}\right)\right), y\right)
$$

Then the sum in (31) can be written as a Stieltjes integral

$$
\int_{i}^{\infty} \operatorname{Erfc}(u) d \Phi(u) \text {. }
$$

Integration by parts now shows that (31) is less than

$$
2 \pi e^{\lambda-1} \Phi(\vartheta)+O\left(\int_{\vartheta}^{\infty} \Phi(u) \exp \left(-u^{2}\right) d u\right) .
$$


6. We now turn to the right-hand side of (29). The value of $\sigma$ will be fixed later, but in any case it will be asymptotically equal to $Z^{-1}=(\log x)^{-1}$. We restrict ourselves to $2 \leqq y \leqq Z^{\Theta}$, for some fixed $\Theta$ with $0<\Theta<\frac{3}{4}$. Assuming that $Z$ is sufficiently large, as we shall always do in what follows without further noticing, we can thus write

$$
N=\sigma^{-1+\eta}
$$

where

$$
\frac{1}{4}<1-\Theta<\eta \leqq 1
$$

Take

$$
T=1 / 2 \sigma^{-1}(\log y)^{-1} .
$$

We split the integral in (29) into three parts, the first part being the integral from $-T$ to $T$ and the others from $T$ to $\infty$ and from $-\infty$ to $-T$. Denote these integrals by $I_{0}, I_{1}, I_{2}$, respectively. Here, of course, $I_{2}$ is the complex conjugate of $I_{1}$. In $I_{0}$ we can use the Laurent series about $s=0$ for the function $F(s)$, the series being convergent in the range of integration, by (15). We infer

$$
I_{0}=P \sigma^{-N} \sum_{n=0}^{\infty} d_{n} \sigma^{n} \int_{-T}^{T} \exp \left(-\lambda w^{2}+i w\right)(1+i w)^{n-N-1} d w .
$$

For $n \geqq N+1$ we use the simple estimate

$$
\left|\int_{-T}^{T} \exp \left(-\lambda w^{2}+i w\right)(1+i w)^{n-N-1} d w\right| \leqq 2 T\left(1+T^{2}\right)^{\frac{1}{2}(n-N-1)}
$$

Since for $0 \leqq n \leqq N$,

$$
\begin{aligned}
& \left|\int_{T}^{\infty} \exp \left(-\lambda w^{2}+i w\right)(1+i w)^{n-N-1} d w\right| \\
& \leqq T^{n-N-1} \int_{T}^{\infty} \exp \left(-\lambda w^{2}\right) d w=\lambda^{-1 / 2} \operatorname{Erfc}\left(T \lambda^{1 / 2}\right) T^{n-N-1},
\end{aligned}
$$

we obtain from (37), (38), (39)

$$
\begin{aligned}
& I_{0}=P \sigma^{-N}\left\{\sum_{n=0}^{N} d_{n} \sigma^{n} J_{N-n+1}\right. \\
& +O\left(\lambda^{-1 / 2} T^{-N-1} \operatorname{Erfc}\left(T \lambda^{1 / 2}\right) \sum_{n=0}^{N}\left|d_{n}\right|(\sigma T)^{n}\right) \\
& \left.\quad+O\left(T^{-N} \sum_{n=N+1}^{\infty}\left|d_{n}\right| \sigma^{n}\left(1+T^{2}\right)^{\frac{1}{2} n}\right)\right\}
\end{aligned}
$$


Let $\varepsilon<\frac{1}{8}$ be an arbitrary fixed positive number and take

$$
\lambda=\sigma^{\min \{2,1+3 \eta\}-\varepsilon} .
$$

By (36) and (41), $\lambda T^{2}>1$, for $\sigma<\sigma(\varepsilon)$, say. (Note that $\sigma \sim Z^{-1}$.) Clearly $\sigma\left(1+T^{2}\right)^{1 / 2}<(\log y)^{-1}$. Hence we can conclude, by (16), that the sum of the $O$-terms in $(40)$ is

$$
\ll T^{-N} \sum_{n=0}^{\infty}\left(\begin{array}{c}
N+n-1 \\
n
\end{array}\right) 2^{-n}=2^{N} T^{-N},
$$

so that

$$
I_{0}=P \sigma^{-N}\left(\sum_{n=0}^{N} d_{n} \sigma^{n} J_{N-n+1}+O\left(2^{N} T^{-v}\right)\right) .
$$

The integral $I_{1}$ is

$$
I_{1}=\int_{T}^{\infty} \sigma \exp \left(-\lambda w^{2}+i w\right) F(\sigma(1+i w)) d w .
$$

The path of integration may be deformed to consist of the line segment from $T$ to $T-i(N-1)$ and the line from $T-i(N-1)$ to $\infty-i(N-1)$. Let the corresponding integrals be $I_{1}^{\prime}$ and $I_{1}^{\prime \prime}$.

By geometrical considerations, it is easy to see that $1-p^{-u-i \sigma T}$ decreases when $u$ increases from $\sigma$ to $\sigma N$, if

$$
p^{-\sigma N}>\cos (\sigma T \log p) \text {. }
$$

The validity of this condition for $p \leqq y$ is easily derived from (35) and (36). Hence on the first path of integration $|f(\sigma(1+i w))|$ takes on its greatest value at the end point and we obtain

$$
\begin{aligned}
\left|I_{1}^{\prime}\right| & <T^{-1} f(\sigma N) \int_{0}^{N-1} \exp \left(-\lambda T^{2}+\lambda u^{2}+u\right) d u \\
& <T^{-1} f(\sigma N) \exp \left(-\lambda T^{2}+\lambda N^{2}+N\right) \\
& <T^{-1} P(\sigma N)^{-N} Q\left(\sigma\left(1+\frac{1}{6} \sigma N \log y\right)\right) \exp \left(-\lambda T^{2}+\lambda N^{2}+N\right),
\end{aligned}
$$

where the last step is easily derived from (13). Using Stirling's formula, observing that $N^{1 / 2} T^{-1}<1$,

$$
\lambda N^{2}=\sigma^{\min \{2 \eta,-1+5 \eta\}-\varepsilon}<\sigma^{\frac{1}{4}-\varepsilon}<1,
$$

and putting

$$
\sigma^{*}=\sigma\left(1+\sigma^{\eta} \log y\right),
$$


we can write this estimate in the following form

$$
\left|I_{1}^{\prime}\right| \ll P \sigma^{-N}(N !)^{-1} Q\left(\sigma^{*}\right) \exp \left(-\lambda T^{2}\right) .
$$

We can deal in a similar way with $I_{1}^{\prime \prime}$. This leads to

$$
\begin{aligned}
\left|I_{1}^{\prime \prime}\right| & <N^{-1} f(\sigma N) \int_{T}^{\infty} \exp \left(-\lambda u^{2}+\lambda N^{2}+N\right) d u \\
& \ll P \sigma^{-N}(N !)^{-1} Q\left(\sigma^{*}\right) \lambda^{-\frac{1}{2}} \quad \operatorname{Erfc}\left(\lambda^{1 / 2} T\right) \\
& \ll P \sigma^{-N-1}(N !)^{-1} Q\left(\sigma^{*}\right) \exp \left(-\lambda T^{2}\right),
\end{aligned}
$$

in virtue of (5), (36), (41). Comparing (45) and (46) we see that the righthand side of (46) also gives an upper bound for $\left|I_{1}\right|$.

7. We shall now state our main theorem:

Theorem 2. We have, uniformly for $2 \leqq y \leqq Z^{\Theta}$ with $0<\Theta<\frac{\mathbf{3}}{\mathbf{4}}$,

$$
\Psi\left(e^{Z}, y\right)=P Z^{N} R\left(\frac{1}{Z}\right)\left(1+O\left(Z^{-\frac{1}{8}+\varepsilon}\right)\right),
$$

where $\varepsilon$ is any positive number and the constant implied by the O-notation only depends on $\Theta$ and $\varepsilon$.

Proof. Consider at first the case $2 \leqq y \leqq Z^{7 / 16}$. It follows from Theorem 1 that then

$$
\Psi\left(e^{Z}, y\right)=P(N !)^{-1} Z^{N}\left(1+O\left(Z^{-\frac{1}{8}}\right)\right) .
$$

On the other hand, by the same procedure that was used to prove (27), we can easily deduce that, for sufficiently large $Z$,

$$
\sum_{n=1}^{N} \frac{\left|d_{n}\right| Z^{-n}}{(N-n) !} \ll(N !)^{-1} y^{2} Z^{-1} \leqq(N !)^{-1} Z^{-\frac{1}{8}},
$$

whence Theorem 2 is true in this case.

We may thus assume that $y>Z^{7 / 16}$. In particular, we may assume that the condition $N \geqq N_{0}(\Theta)$, introduced in section 4 , is satisfied.

We begin with the upper bound of $\Psi\left(e^{Z}, y\right)$. Take

$$
\vartheta=\sigma^{-1 / 2 \varepsilon}
$$

and define $\sigma$ by

$$
\sigma^{-1}\left(1-2 \lambda-2 \vartheta \lambda^{1 / 2}\right)=Z
$$

such a choice of $\sigma$ obviously being possible with $\sigma$ asymptotically equal to $Z^{-1}$. Clearly

$$
1-\vartheta^{-1} \exp \left(-\vartheta^{2}\right)=1+O\left(Z^{-1}\right)
$$


Combining (29), (30), (42), (45), (46), we have

$$
\begin{aligned}
& 2 \pi e^{\lambda-1}\left(1+O\left(Z^{-1}\right)\right) \Psi\left(e^{Z}, y\right) \leqq P \sigma^{-N}\left\{\sum_{n=0}^{N} d_{n} \sigma^{n} J_{N-n+1}\right. \\
& \left.+O\left(2^{N} T^{-N}\right)+O\left(Z(N !)^{-1} Q\left(\sigma^{*}\right) \exp \left(-\lambda T^{2}\right)\right)\right\} .
\end{aligned}
$$

Applying (12), we replace the $J_{N-n+1}:$ s by $(1+3 \lambda N)^{N}((N-n) !)^{-1} J_{1}$, so that the sum becomes $(1+3 \lambda N)^{N} J_{1} R(\sigma)$. Here, by (43),

$$
(1+3 \lambda N)^{N}<\exp \left(3 \lambda N^{2}\right)=1+O\left(Z^{-\frac{1}{4}+\varepsilon}\right) .
$$

Now (27) shows that the influence of the possible negative terms is compensated simply by adding an error term $O\left(y^{-1}(N !)^{-1}\right)$. Replacing $\sigma^{-N}$ in (49) by $Z^{N}$ we must add an extra factor

$$
\left(1-2 \lambda-2 \vartheta \lambda^{1 / 2}\right)^{-N} .
$$

The logarithm of this is, by (43), (47), (48),

$$
\ll \vartheta \lambda^{1 / 2} N<Z^{-\frac{1}{8}+\varepsilon} .
$$

Clearly, by (9),

$$
J_{1}=2 \pi e^{\lambda-1}\left(1+O\left(Z^{-1}\right)\right) .
$$

It follows from these considerations that

$$
\begin{aligned}
& \Psi\left(e^{Z}, y\right) \leqq P Z^{N}\left\{R(\sigma)+O\left(y^{-1}(N !)^{-1}\right)+O\left(2^{N} T^{-N}\right)\right. \\
& \left.+O\left(Z(N !)^{-1} Q\left(\sigma^{*}\right) \exp \left(-\lambda T^{2}\right)\right)\right\}\left(1+O\left(Z^{-\frac{1}{8}+\varepsilon}\right)\right) .
\end{aligned}
$$

It is easy to show that (23) and (28) imply

$$
(N !)^{-1} \ll R(\sigma) \text {. }
$$

Hence the first error term on the right-hand side of (50) can be omitted and so can also the second one, since

$$
N ! 2^{N} T^{-N} \ll N^{1 / 2}\left(\frac{4 N \log y}{e Z}\right)^{N} .
$$

To estimate the third error term we use (28) with $t=\sigma^{*}$ (an admissible choice by (25)). It follows that

$$
Q\left(\sigma^{*}\right)(N !)^{-1}<\left(R\left(\sigma^{*}\right)+2 y^{-1}(N !)^{-1}\right) \exp \left(2 n_{0}^{2} N^{-1}\right),
$$

where $n_{0}=n_{0}\left(\sigma^{*}\right)$ is defined by (26). We break the sum $R\left(\sigma^{*}\right)$ into two parts, the first part consisting of those terms with $0 \leqq n \leqq n_{0}$. The second part is $\left\langle y^{-1}(N !)^{-1}\right.$, by (27). In the first part we have, by (44),

$$
\sigma^{* n} \leqq \sigma^{n}\left(1+\sigma^{\eta} \log y\right)^{n_{0}}<\sigma^{n} \exp \left(\sigma^{\eta} n_{0} \log y\right) .
$$


Hence the first part may be replaced by this factor times $R(\sigma)$ plus an error term $y^{-1}(N !)^{-1}$. We thus arrive at

$$
Q\left(\sigma^{*}\right)(N !)^{-1}<\left(R(\sigma)+4 y^{-1}(N !)^{-1}\right) \exp \left(2 n_{0}^{2} N^{-1}+\sigma^{\eta} n_{0} \log y\right) .
$$

Since

$$
\lambda T^{2}=\frac{1}{4} \sigma^{\min \{0,-1+3 \eta\}-\varepsilon}(\log y)^{-2} \geqq \sigma^{\min \{0,-1+3 \eta\}-1 / 2 \varepsilon},
$$

we obtain, by (26) and (51), for the third $O$-term on the right-hand side of (50) the estimate

$$
\begin{gathered}
\ll Z R(\sigma) \exp \left(-\sigma^{\min \{0,-1+3 \eta\}-1 / 2 \varepsilon}+2 e^{2} \log ^{2} y \sigma^{\min \{1-\eta,-1+3 \eta\}}\left(1+\sigma^{\eta} \log y\right)^{2}\right. \\
\left.+e \log ^{2} y \sigma^{\min \{\eta,-1+3 \eta\}}\left(1+\sigma^{\eta} \log y\right)\right) \\
<Z R(\sigma) \exp \left(-Z^{\frac{1}{4}}\right)^{2} .
\end{gathered}
$$

Hence also this term may be omitted. Finally, we replace $\sigma$ by $Z^{-1}$ in $R(\sigma)$. Since $\sigma<Z^{-1}$, this morely increases the right-hand side of $(50)$, the possible negative terms being again controlled by an error term which in turn has no affect to the final formula. This proves that the required upper estimate is valid.

We only sketch the proof of the lower estimate, since the argument is completely analogous and in some respects even simpler. We define $\vartheta$ by (47) as above, but this time we determine $\sigma$ from

$$
\sigma^{-1}\left(1-2 \lambda+2 \vartheta \lambda^{1 / 2}\right)=Z
$$

This is again clearly possible with $\sigma$ asymptotically equal to $Z^{-1}$. We then use the upper bound (34) of the left-hand side of (29) in order to obtain an inequality of the same type as (49), but with the sign of inequality reversed. Next we use (11), and the proof proceeds as above. The only essential difference is the $O$-term in (34). In order to estimate $\Phi(u)$ (defined by (33)) for $u \geqq \vartheta$, we use that part of our theorem which we already have proved. Hence, for $u \geqq \vartheta$,

$$
\Phi(u) \ll P \sigma^{-N}\left(1-2 \lambda+2 u \lambda^{1 / 2}\right)^{N} R\left(\sigma\left(1-2 \lambda+2 u \lambda^{1 / 2}\right)^{-1}\right) .
$$

Using the same reasoning as above, we see that in the $R$-expression $u$ may be replaced by $\vartheta$. Thus, by (53),

$$
\Phi(u) \ll P Z^{N} R\left(Z^{-1}\right)\left(1+2 u \hat{\imath}^{1 / 2}\right)^{N},
$$

so that 


$$
\int_{\vartheta}^{\infty} \Phi(u) \exp \left(-u^{2}\right) d u \ll P Z^{N} R\left(Z^{-1}\right) \int_{\vartheta}^{\infty} \exp \left(-u^{2}+2 u \lambda^{1 / 2} N\right) d u .
$$

By (43), the integral on the right-hand side of $(54)$ is $\ll \operatorname{Erfc}(\vartheta-1)$. Clearly, therefore, also the error term (54) is harmless. This concludes the proof of Theorem 2.

UnIVERstTy OF TuRKu

Turku, Finland

\section{References}

[1] N. G. DE Bruijn: On the number of positive integers $\leqq x$ and free of prime factors $>y$. - Indag. Math. XIII (1951), 50-60.

[2] -»- On the number of positive integers $\leqq x$ and free of prime factors $>y$, II. - Indag. Math. XXVIII (1966), 239-247.

[3] P. Erdös and J. H. van Lint: On the number of positive integers $\leqq x$ and free of prime factors. $>y$. - Simon Stevin 40 (1966), $73-76$.

[4] A. E. Ingham: A Tauberian theorem for partitions. - Ann. of Math. 42 (1941), $1075-1090$.

[5] A. I. Vinogradov: On numbers with small prime divisors (Russian). - Dokl. Akad. Nauk. SSSR 109 (1956), 683-686. 